\title{
LINE CAPACITANCE AND IMPEDANCE OF COPLANAR-STRIP WAVEGUIDES ON SUBSTRATES WITH MULTIPLE DIELECTRIC LAYERS
}

\author{
S. Gevorgian ${ }^{1,2}$ and H. Berg ${ }^{1}$ \\ ${ }^{1}$ Department of Microelectronics, Chalmers University of Technology, Gothenburg, Sweden \\ ${ }^{2}$ Ericsson Microwave Systems, Moelndal, Sweden \\ email:spartak@ep.chalmers.se
}

\begin{abstract}
Closed form formulas for the basic parameters of Coplanar-Strip line on a finite thickness substrate are reviewed. New, improved formulas are derived using conformal mapping technique.
\end{abstract}

\section{INTRODUCTION}

Coplanar-strip (CPS) waveguides have almost the same advantages over microstrip lines as Coplanar Waveguides (CPW). Additionally, symmetric CPS is an inherently balanced line, i.e. the currents in the strips are equal and opposite - a feature required in a number of microwave applications, such as balanced mixers, amplifiers, antennas etc. In modern microwave integrated circuits the substrates usually consist of multiple layers. Nevertheless, there is a lack in reliable close form CAD oriented formulas for characterization of these lines on multiple dielectric layer substrates. The results for multilayer substrate CPS given in [1] are based on formulas derived for a single layer substrate CPS [2], Fig.1a. It has been indicated in [2], that the conformal mapping based analytic expression for the impedance of finite thick substrate CPS, first reported by Fouad-Hanna [3], become useless where the thickness of the substrate tends to zero. To overcome this problem Ghione and Naldi [2] proposed to use the formulas of the effective dielectric constant of CPW [4] also for CPS, making use of the principle of complementarity [5]. When comparing the results in [2] and [3] a question arises why the conformal mapping technique successfully used for the CPW does not work for the CPS? It turns out that in the original analysis Fouad-Hanna used not an adequate transformation leading to above mentioned [2] problems. Furthermore, the principle of complementarity may be used where the thickness of the substrate is infinite [2], and the dielectric constant of substrate is small. Strictly speaking, it is correct for CPS and CPW without substrate [5]. Hence the results presented in [3] and [4] may be used in limiting cases.

An improved analysis for the finite extent substrate CPS is given in this work, which is then used for a CPS on a substrate with an additional dielectric layer between the substrate and the strips, Fig.1b. The results obtained in this work are in a good agreement with the available experimental data and full wave analysis and could be easily extended to CPS on multilayer substrate CPS.

\section{SINGLE LAYER SUBSTRATE CPS}

The sequence of conformal transformations for a finite thickness substrate CPS is shown in Fig.1. The substrate is assumed to have dielectric constant $\varepsilon$, and thickness $h$. The width of the strips is s, and the spacing between them is $2 g$, Fig. 1 . First, we evaluate the partial capacitance due to dielectric slab with an equivalent dielectric constant $(\varepsilon-1)$ [4], [6], [7]. The symmetry plane at $x=0$ is regarded as an electric wall, while the dielectric/air interfaces are approximated by magnetic walls. Then the capacitance between the strips is the capacitance of series connected between the strips and the electric wall capacitors, Fig.1a. In the first step the semi-infinite right hand side of the dielectric strip with a thickness $h$ and equivalent dielectric permittivity $(\varepsilon-1)$ in the Z-plane, Fig1a, is mapped on a lower part of $T$-plane, Fig.2a, using transformation $t=\cosh ^{2}(\pi \mathrm{Z} / 2 h)$. This mapping results in the following vortex coordinates in $T$-plane: $t_{1}=1 ; t_{2}=\cosh ^{2}(\pi g / 2 h) ; t_{3}=\cosh ^{2}[\pi(s+g) / 2 h) ; t_{4}=t_{5}=\infty ; t_{6}=0$. The lower part of the $T$-plane is mapped on a rectangle in $W$-plane, shown in Fig.2b, using Schwartz-Christoffel 
transformation. For coordinates given above $\left(t>t_{3}>t_{2}>t_{1}>t_{6}\right)$ the modulus of the complete elliptic integral [8] (p.72, 1.2.23) may be transformed to

$$
k=\frac{\tanh \left(\frac{\pi g}{2 h}\right)}{\tanh \left(\frac{\pi(s+g)}{2 h}\right)}
$$

From the last transformation $w_{l}=K(k)+j K\left(k^{\prime}\right)$, Fig.2b. Then the capacitance of the parallel-plate capacitor, Fig.2b, is:

$$
C=\varepsilon_{o}(\varepsilon-1) \frac{K\left(k^{\prime}\right)}{K(k)}
$$

$K(k)$ and $j K\left(k^{\prime}\right)$ complete elliptic integrals of the first kind with $k^{\prime}=\sqrt{ }\left(1-k^{2}\right)$. A similar capacitance is due to the left-hand side of Fig.1a, hence the total partial capacitance due the substrate is:

$$
C_{s}=\varepsilon_{o}(\varepsilon-1) \frac{K\left(k^{\prime}\right)}{2 K(k)}
$$

The partial capacitance between the two strips (air above and below), Fig.1a, in the absence of the substrate is derived from (3) at $h=\infty$ limit, assuming $(\varepsilon-1)=1$ :

$$
C_{a}=\varepsilon_{o} \frac{K\left(k_{o}^{\prime}\right)}{K\left(k_{o}\right)},
$$

where

$$
k_{o}=\frac{g}{s+g}, \quad k_{o}^{\prime}=\sqrt{1-k_{o}^{2}}
$$

Finally, the total line capacitance, Fig.1a, is $C=C_{s}+C_{a}$

$$
C=\varepsilon_{o} \varepsilon_{e f f} \frac{K\left(k_{o}^{\prime}\right)}{K\left(k_{o}\right)}
$$

with an effective dielectric constant and filling factor defined as: $\mathcal{E}_{\text {eff } 1}=1+(\mathcal{\varepsilon}-1) q$,

$$
q=\frac{1}{2} \frac{K\left(k^{\prime}\right)}{K(k)} \frac{K\left(k_{o}\right)}{K\left(k_{o}^{\prime}\right)}
$$

The impedance of a finite thick substrate CPS is

$$
Z=\frac{120 \pi}{\sqrt{\varepsilon_{\text {eff }}}} \frac{K\left(k_{o}\right)}{K\left(k_{o}^{\prime}\right)}
$$

\section{TWO LAYER SUBSTRATE CPS}

In this case, we assume air above the strips and below the substrate, Fig.1b. It is assumed that the dielectric permittivity of the additional layer is larger than the dielectric permittivity of the substrate. The total line capacitance of this CPS may be written as a sum of partial capacitances, $C=C_{a}+C_{l}+C_{2}$, 
where $C_{a}$ is the capacitance between the strips in the absence of dielectric layers (4). $C_{1}$ and $C_{2}$ are the partial capacitancies of the dielectric layers with equivalent dielectric constants $\left(\varepsilon_{1}-1\right)$ and $\left(\varepsilon_{2}-\varepsilon_{1}\right)$, and thickness $h_{1}$ and $h_{2}$ respectively [6]. These capacitances are found by using a procedure, which has led to (3) in the previous section. The resulting capacitance of a two layer substrate CPS is

$$
C=\varepsilon_{o} \varepsilon_{e f f} \frac{K\left(k_{o}^{\prime}\right)}{K\left(k_{o}^{\prime}\right)}
$$

with an effective dielectric constant $\varepsilon_{\text {eff } 2}=1+\left(\varepsilon_{1}-1\right) q_{1}+\left(\varepsilon_{2^{-}} \varepsilon_{1}\right) q_{2}$. The filling factors, associated modulus of the elliptic integrals and the impedance of this CPS are given as:

$$
\begin{aligned}
& q_{i}=\frac{1}{2} \frac{K\left(k_{i}^{\prime}\right)}{K\left(k_{i}\right)} \frac{K\left(k_{o}\right)}{K\left(k_{o}^{\prime}\right)} ; \quad i=1,2 \\
& k_{i}=\frac{\tanh \left(\frac{\pi g}{2 h_{i}}\right)}{\tanh \left(\frac{\pi(s+g)}{2 h_{i}}\right)} ; \quad i=1,2 \\
& Z=\frac{120 \pi}{\sqrt{\varepsilon_{\text {eff } 2}}} \frac{K\left(k_{o}\right)}{K\left(k_{o}^{\prime}\right)}
\end{aligned}
$$

\section{DISCUSSIONS AND NUMERICAL RESULTS}

The difference between the formulas above and those given in [2], [3], first of all, is in the modulus $k$. In (1) $k$ is given as a ratio of tanh functions while Fouad-Hanna [3] and Ghione and Naldi [2] use a ratio of $\sinh$ functions. For $g / h$ and $(s+g) / h \rightarrow 0$ limit $k$ given by (1) and by [2], [3] tend to $k_{o}(5)$. For $g / h$ and $(s+g) / h \rightarrow \infty$ limit $k$ given by (1) tend to $k=1$, while $k$ given by sinh functions [4], [3] tend to $k=0$. The above limits correspond to $h \rightarrow \infty$ and $h \rightarrow 0$. Strictly speaking the at $h \rightarrow \infty$ limit the principle of complementarity, used in [2], may be applied for small dielectric permittivity of the substrate, if the magnetic wall approximation at air/dielectric interfaces (between the strips) is not strongly violated. In general, away from these limits the principle of complementarity is violated, and in some cases one may expect inaccuracies in the effective dialectic constant $\varepsilon_{\text {eff }}$, line capacitance $C$ and impedance $Z$ where they are computed using the results presented in [2]. For a CPS with an infinite thickness substrate, $h \rightarrow \infty$ limit, $k \approx k_{o}$, from (8) q=1/2, leading to will known limits for the effective dielectric constant, $\varepsilon_{\text {eff }}=(\mathcal{E}+1) / 2$, and line capacitance. A good agreement, at low frequencies, is also found with the results of full wave analysis given in [9].

Proposed formulas are especially useful for CPS with extremely high dielectric permittivity, such as ferroelectrics, where most of the commercially available software (i.e. Momentum) fail to work. The line parameters for a CPS with a ferroelectric layer $\left(h_{2}=1.0 \mu \mathrm{m}, \varepsilon=100, h_{1}=400 \mu \mathrm{m}, \mathrm{s}=125 \mu \mathrm{m}\right)$ computed using (7) and Partial Element Equivalent Circuit (PEEC) method [9] at $10 \mathrm{GHz}$ are shown in Table 1.

\section{CONCLUSIONS}

Improved closed form formulas for the basic parameters of one and two layer substrate CPS have been derived. The dielectric constant of the layer between the strips and substrate should not be smaller then the dielectric constant of the substrate, $\varepsilon_{2}>\varepsilon_{1}$. For $\varepsilon_{2}<\varepsilon_{1}$ the magnetic wall approximation at the dielectric/dielectric interfaces is violated [11], and the accuracy of above approximation is very poor. 


\section{REFERENCES}

1. E. Chen and S.Y. Chu, "Characteristics of Coplanar Transmission Lines on Multilayer Substrates: Modeling and experiment", IEEE Trans. Microwave Theory Techn., Vol.45, pp.939-945, 1997

2. G. Ghione, and C. Naldi, "Analytical Formulas for Coplanar Lines in Hybrid and Monolithic MICs" Electron. Letter., Vol.20, pp.179-181, 1984

3. V. Fouad-Hanna, "Finite Boundary Corrections to Coplanar Stripline Analysis", Electron. Letters., Vol.16, pp. 604-606, 1980

4. C. Veyres, and V. Fouad-Hanna, "Extension of the Application of Conformal Mapping Technique to Coplanar Lines with Finite Dimensions", Int. J. Electron., Vol.48, pp.47-56, 1980

5. G. H. Owing, and R. King, "Complementarity in Study of Transmission Lines", IRE Trans. Microw. Theory Techn., March, pp.172-181, 1960

6. Gevorgian S. S., "Basic Characteristics of Two Layered Coplanar Waveguides", Electronics Letters,Vol.30, N15, pp.1236-1237, 1994

7. G. Ghione, and C. U. Naldi, "Coplanar Waveguides for MMIC Applications: Effect of Upper Shielding, Conductor Backing, Finite-Extent Ground Planes, and Line-to-Line Coupling”, IEEE Trans. Microwave Theory Techn., Vol.35, pp.260-267, 1987

8. A. P Prudnikov, Yu. A Brychkov, Integrals and Serieses, Gordon and Breach, 1986

9. K. M. Rahman, and C. Nguyen, "Full-Wave Analysis of Coplanar-Strips Considering the Finite Metallization Thickness", IEEE Trans., Vol.42, pp.2177-2179, 1994

10. H. Berg, "A Simple Method for Evaluation of Line Capacitances in MMICs on Multilayer Substrates Including Losses in Dielectrics", Submitted to EuMC'2001.

11. E. Carlsson, and S. Gevorgian "Conformal Mapping of the Field and Charge Distributions in Multilayered Substrate CPWs" IEEE Trans. Microwave Theory Techn., Vol. 47, pp. 1544-1552,

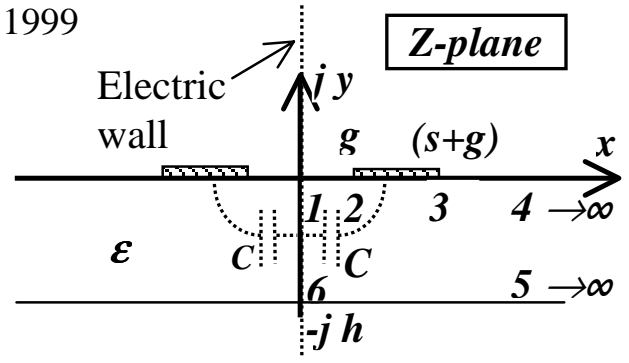

Fig.1 Single (a) and double (b) layer substrate CPS

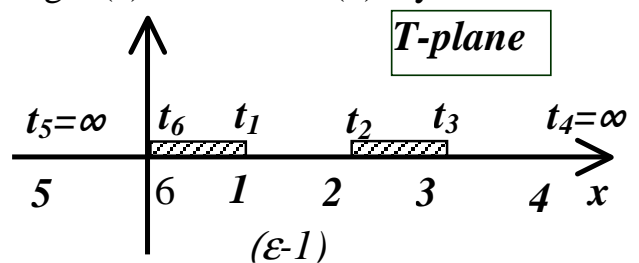

a)

Fig.2 Sequence of conformal transformations

Table 1 CPS parameters computed by conformal mapping and PEEC

\begin{tabular}{|l|l|l|l|l|}
\hline \multirow{2}{*}{} & \multicolumn{3}{|l|}{$2 g=2 \mu \mathrm{m}$} & \multicolumn{2}{l|}{$2 g=4 \mu \mathrm{m}$} \\
\cline { 2 - 5 } & This work & {$[9]$} & This work & {$[9]$} \\
\hline $\mathrm{C} \mathrm{pF} / \mathrm{m}$ & 491 & 517 & 355 & 373 \\
\hline$\varepsilon_{\text {eff } 2}$ & 14.0 & 14.7 & 11.4 & 12.0 \\
\hline$Z, \mathrm{Ohm}$ & 25.4 & 24.8 & 31.6 & 31.0 \\
\hline
\end{tabular}

Fig.3 Impedance of CPS vs. substrate thickness

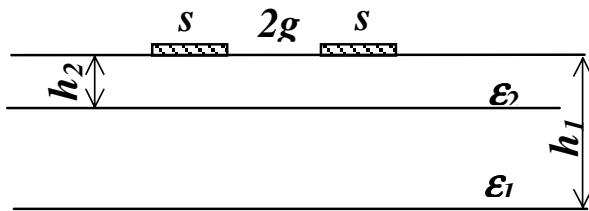

b)

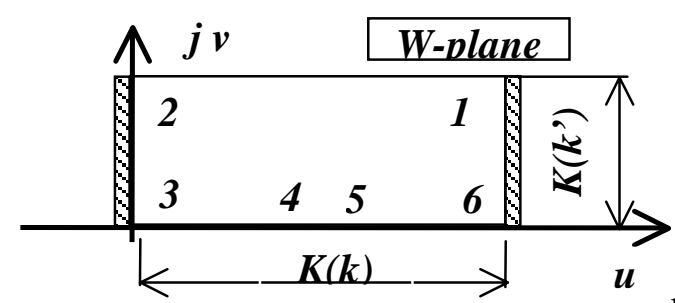

b)

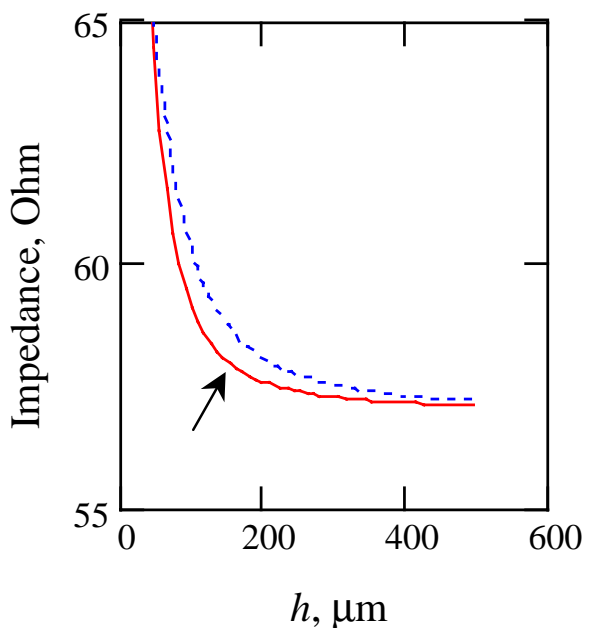

